\title{
Young Massive Clusters
}

\author{
Donald F. Figer \\ Chester F. Carlson Center for Imaging Science \\ Rochester Institute of Technology \\ Rochester, NY 14623-5604 \\ email: figer@cis.rit.edu
}

\begin{abstract}
Over the past ten years, there has been a revolution in our understanding of massive young stellar clusters in the Galaxy. Initially, there were no known examples having masses $>10^{4}$, yet we now know that there are at least a half dozen such clusters in the Galaxy. In all but one case, the masses have been determined through infrared observations. Several had been identified as clusters long ago, but their massive natures were only recently determined. Presumably, we are just scratching the surface, and we might look forward to having statistically significant samples of coeval massive stars at all important stages of stellar evolution in the near future. I review the efforts that have led to this dramatic turn of events and the growing sample of young massive clusters in the Galaxy.
\end{abstract}

Keywords. open clusters and associations: general - stars: early-type

\section{Introduction}

Massive stellar clusters are the birthplaces of massive stars. They are also the places where many massive stars reside all of their lives, having little time to wander before exploding as supernovae. The astrophysical importance of massive clusters largely derives from their content of massive stars, objects that have extraordinary effects on their surroundings. Indeed, massive stars are key ingredients and probes of astrophysical phenomena on all size and distance scales, from individual star formation sites, such as Orion, to the early Universe during the age of reionization when the first stars were born. As ingredients, they control the dynamical and chemical evolution of their local environs and individual galaxies through their influence on the energetics and composition of the interstellar medium. They likely play an important role in the early evolution of the first galaxies, and there is evidence that they are the progenitors of the most energetic explosions in the Universe, seen as GRBs. As probes, they define the upper limits of the star formation process and their presence may end further formation of nearby lower mass stars and planets.

Despite the importance of massive stars, and the clusters in which they reside, no truly massive clusters were known to exist in the Galaxy before about ten years ago, when the Arches and Quintuplet clusters were identified as being at least that massive (Figer et al. 1999b). Since then, a number of efforts have led to the identification of about a half dozen more such massive clusters in the Galaxy.

The evolution of massive stars is difficult to study because many of the most important phases are short. To date, one of the most effective techniques to overcome this problem is to identify massive clusters at ages when its most massive stars are largely in a single phase of evolution., We see this in the Arches cluster (the most massive stars, i.e. hydrogen burning Wolf-Rayet stars on the main sequence), rhe Central Cluster (Ofpe/WN9 stars), Westerlund 1 (Wolf Rayet stars), and the Scutum red supergiant clusters (RSGs). 
Table 1. Properties of massive clusters in the Galaxy ${ }^{a}$

\begin{tabular}{|c|c|c|c|c|c|c|c|c|c|c|c|c|}
\hline Cluster & $\begin{array}{r}\log (M) \\
\mathrm{M}_{\odot}\end{array}$ & $\begin{array}{r}\text { Radius } \\
\text { pc }\end{array}$ & $\begin{array}{r}\log (\rho) \\
\mathrm{M}_{\odot} \mathrm{pc}^{-3}\end{array}$ & $\begin{array}{l}\text { Age } \\
\text { Myr }\end{array}$ & $\begin{array}{r}\log (L) \\
\mathrm{L}_{\odot}\end{array}$ & $\begin{array}{r}\log (Q) \\
\mathrm{s}^{-1}\end{array}$ & $\mathrm{OB}$ & YSG & RSG & LBV & WN & $\mathrm{WC}$ \\
\hline Westerlund $1^{b}$ & 4.7 & 1.0 & 4.1 & $4-6$ & $\ldots$ & $\ldots$ & $\ldots$ & 6 & 4 & 2 & 16 & 8 \\
\hline $\mathrm{RSGC} 2^{c}$ & 4.6 & 2.7 & 2.7 & $14-21$ & $\ldots$ & $\ldots$ & 0 & 0 & 26 & 0 & 0 & 0 \\
\hline $\mathrm{RSGC}^{d}$ & 4.5 & 1.3 & 3.5 & $10-14$ & $\ldots$ & $\ldots$ & 1 & 1 & 14 & 0 & 0 & 0 \\
\hline Quintuplet ${ }^{e}$ & 4.3 & 1.0 & 3.2 & $4-6$ & 7.5 & 50.9 & 100 & 100 & 1 & 2 & 6 & 13 \\
\hline $\operatorname{Arches}^{f}$ & 4.3 & 0.19 & 5.6 & $2-2.5$ & 8.0 & 51.0 & 160 & 0 & 0 & 0 & 6 & 0 \\
\hline Center $^{g}$ & 4.3 & 0.23 & 5.6 & $4-7$ & 7.3 & 50.5 & 100 & 0 & 4 & 1 & 18 & 12 \\
\hline NGC $3603^{h}$ & 4.1 & 0.3 & 5.0 & $2-2.5$ & $\ldots$ & $\ldots$ & 60 & 0 & 0 & 0 & 3 & 0 \\
\hline Trumpler $14^{i}$ & 4.0 & 0.5 & 4.3 & $<2$ & $\ldots$ & $\ldots$ & 31 & $\ldots$ & $\ldots$ & $\ldots$ & $\ldots$ & $\ldots$ \\
\hline Westerlund $2^{j}$ & 4.0 & 0.8 & 3.7 & $1.5-2.5$ & $\ldots$ & $\ldots$ & $\ldots$ & $\ldots$ & $\ldots$ & $\ldots$ & 2 & .. \\
\hline $\mathrm{Cl} 1806-20^{k}$ & 3.8 & 0.8 & 3.5 & $4-6$ & $\ldots$ & $\ldots$ & 5 & 0 & $\ldots$ & 1 & 2 & 2 \\
\hline
\end{tabular}

${ }^{a}$ An ellipsis has been entered in cases where data are not reliable or available. "M" is the total cluster mass in all stars extrapolated down to a lower-mass cutoff of $1 \mathrm{M}_{\odot}$, assuming a Salpeter IMF slope and an upper mass cutoff of $120 \mathrm{M}_{\odot}$ (unless otherwise noted) "Radius" gives the average projected separation from the centroid position. " $\rho$ " is $\mathrm{M}$ divided by the volume. This is probably closer to the central density than the average density because the mass is for the whole cluster while the radius is the average projected radius. "Age" is the assumed age for the cluster. "Luminosity" gives the total measured luminosity for observed stars. "Q" is the estimated Lyman continuum flux emitted by the cluster. ${ }^{b}$ Figer et al. (2006). ${ }^{c}$ Davies et al. (2007).

${ }^{d}$ Figer et al. (2006). ${ }^{e}$ Figer et al. (1999b). ${ }^{f}$ Mass estimates have been made based upon the number of stars having $M_{\text {initial }}>20 \mathrm{M}_{\odot}$ given in Figer et al. $(1999 \mathrm{~b})$ and the mass function slope in Kim et al. (2006). The age, luminosity and ionizing flux are from Figer et al. (2002). ${ }^{g}$ Krabbe et al. (1995). The mass, M has been estimated by assuming that a total $10^{3.5}$ stars have been formed. The age spans a range covering an initial starburst, followed by an exponential decay in the star formation rate. ${ }^{h}$ Harayama (2007). ${ }^{i}$ Harayama (2007). ${ }^{j}$ Harayama (2007). ${ }^{k}$ Figer et al. (2005).

We are on the cusp of a revolution in massive stellar cluster research, as the identified sample is likely the "tip of the iceberg." In the next ten years, we can expect to identify perhaps a factor of ten more massive clusters in the Galaxy than are currently known. With this sample, we can expect to routinely address many of the long-pursued questions in massive star research to determine, for example: 1) the most massive star that can form, 2) the binary frequency of massive stars, 3) the properties of massive stellar winds, e.g. clumping, 4) the evolutionary sequence of massive stars, and 5) the end states of massive stars. Indeed, Barbosa \& Figer (2004) summarize many interesting questions that might be addressed with future studies of massive stars.

In this paper, I review the Galactic sample of massive stellar clusters $\left(M>10^{4} M_{\odot}\right)$, the efforts that led to their discovery, and the identification of a stellar upper mass limit. Finally, I speculate on the hidden population of massive stellar clusters in the Galaxy that will likely be revealed in the near future.

\section{The sample of massive young clusters}

There are approximately ten known Galactic clusters with masses $\gtrsim 10^{4} \mathrm{M}_{\odot}$, with three being located within the central $50 \mathrm{pc}$ of the Galactic center. Table 1 gives the known properties of these clusters in a list that is ordered with decreasing mass. In some cases, the properties are poorly determined, in which case the entries are filled with an ellipsis. The most massive, Westerlund 1 , has a mass of $\approx 50,000 \mathrm{M}_{\odot}$. The densest are in the Galactic center, with the Arches cluster having a density of $\approx 300,000 \mathrm{M}_{\odot} \mathrm{pc}^{-3}$. While most are quite young, the RSG (red supergiant) clusters are distinctly older. Presumably, the table reflects an observational bias in that younger clusters are generally more concentrated, and thus easier to identify as clusters.

In the following, I review some of the clusters with more well-established characteristics. 


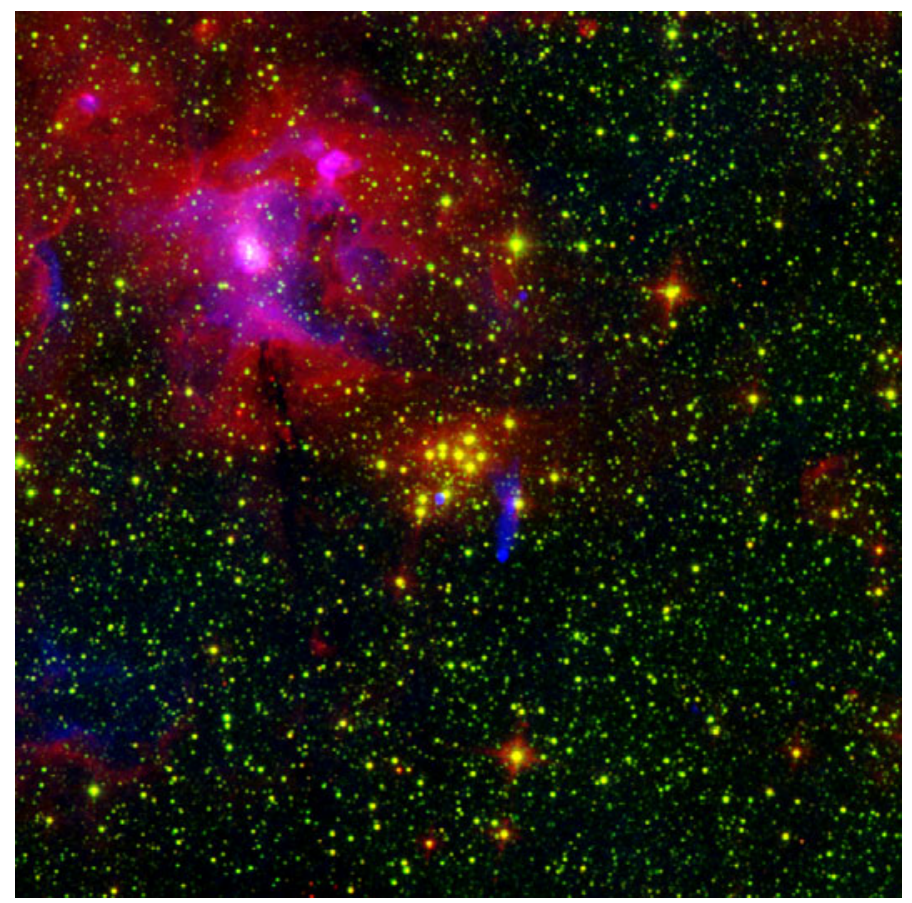

Figure 1. GLIMPSE image of RSGC1. W42, to the upper left, is an unrelated star formation region along the line of sight.

\subsection{Westerlund 1}

Westerlund 1 is the most massive young cluster known in the Galaxy (Clark et al. 2005; Negueruela \& Clark 2005; Skinner et al. 2006; Groh et al. 2006; Crowther et al. 2006; Brandner et al. 2008). Given its age of $\approx 4 \mathrm{Myr}$, it contains more evolved massive stars than any other cluster in the Galaxy, including half the known population of yellow supergiants and the most WR stars. The cluster contains a magnetar, a highly magnetized neutron star that may be descended from a particularly massive progenitor (Muno et al. 2006). Oddly, the massive stellar content in Westerlund 1 has only been recently revealed, more than 40 years after the cluster's discovery.

\subsection{Red Supergiant clusters}

Figer et al. (2006), Davies et al. (2007), and Davies et al. (2008), identified two massive clusters in the red supergiant (RSG) phase, the first containing 14 (see Figure 1) and the second containing 26 RSGs, or collectively about $20 \%$ of all such stars known in the Galaxy. The inferred cluster masses are $\approx 3 \times 10^{4} \mathrm{M}_{\odot}$ for the former, and $\approx 4 \times 10^{4} \mathrm{M}_{\odot}$ for the latter. Interestingly, the two clusters are near each other, located within 1 degree on the sky and within $1 \mathrm{kpc}$ along the line of sight at the base of the Scutum-Crux arm. The first cluster was first identified as a candidate cluster in the Bica et al. (2003) catalog, and some of the stars in the second cluster had already been identified as RSGs in Stephenson (1990) and Nakaya et al. (2001). Just like in the case of Westerlund 1, we see that a cluster once thought to be of relatively low mass can turn out to be quite massive on further inspection. Perhaps there are more massive clusters amongst the many clusters that have already been identified. 


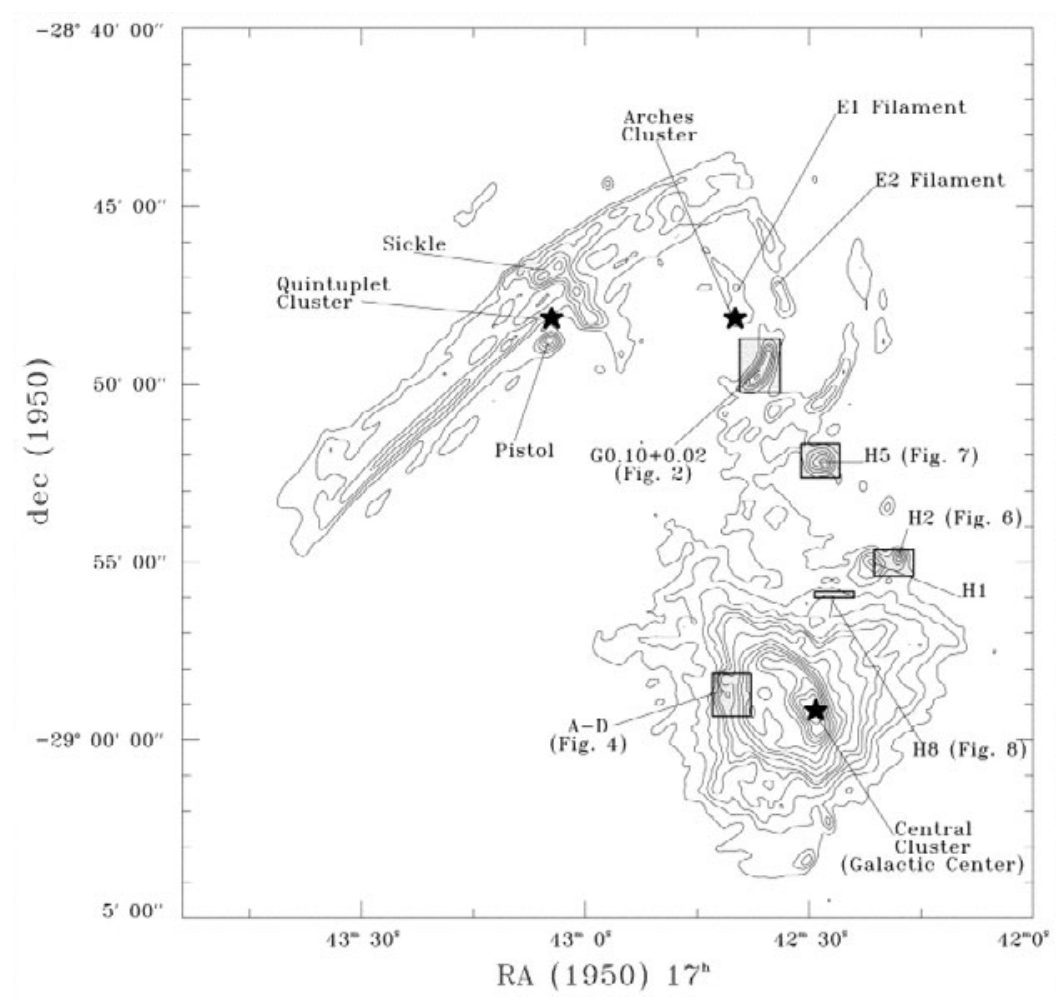

Figure 2. Radio emission from the GC region at $6 \mathrm{~cm}$, adapted by Cotera et al. (1999) from Yusef-Zadeh \& Morris (1987). The star symbols represent the three massive clusters.

\subsection{Central cluster}

The Central cluster resides in the central parsec of the Galaxy and contains many massive stars formed in the past 10 Myr (Becklin et al. 1978; Rieke, Telesco, \& Harper 1978; Lebofsky, Rieke, \& Tokunaga 1982; Forrest et al. 1987; Allen, Hyland, \& Hillier 1990; Krabbe et al. 1991; Najarro et al. 1994; Krabbe et al. 1995; Najarro 1995; Blum, Sellgren, \& Depoy 1995b; Genzel et al. 1996; Najarro et al. 1997). There are at least 80 massive stars in the Central cluster (Eisenhauer et al. 2005), including $\approx 50$ OB stars on the main sequence and 30 more evolved massive stars (see Figure 3). These young stars appear to be confined to two disks (Genzel et al. 2003; Tanner et al. 2006). There is also a tight collection of a dozen or so B stars (the "s" stars) in the central arcsecond, highlighted in the small box in the figure. The formation of so many massive stars in the central parsec remains as much a mystery now as it was at the time of the first infrared observations of the region. Most recently, this topic has largely been supplanted by the even more improbable notion that star formation can occur within a few thousand AU of the supermassive black hole. See Figer (2008), and references therein, for a review of massive star formation and the "s" stars in the Galactic center. 


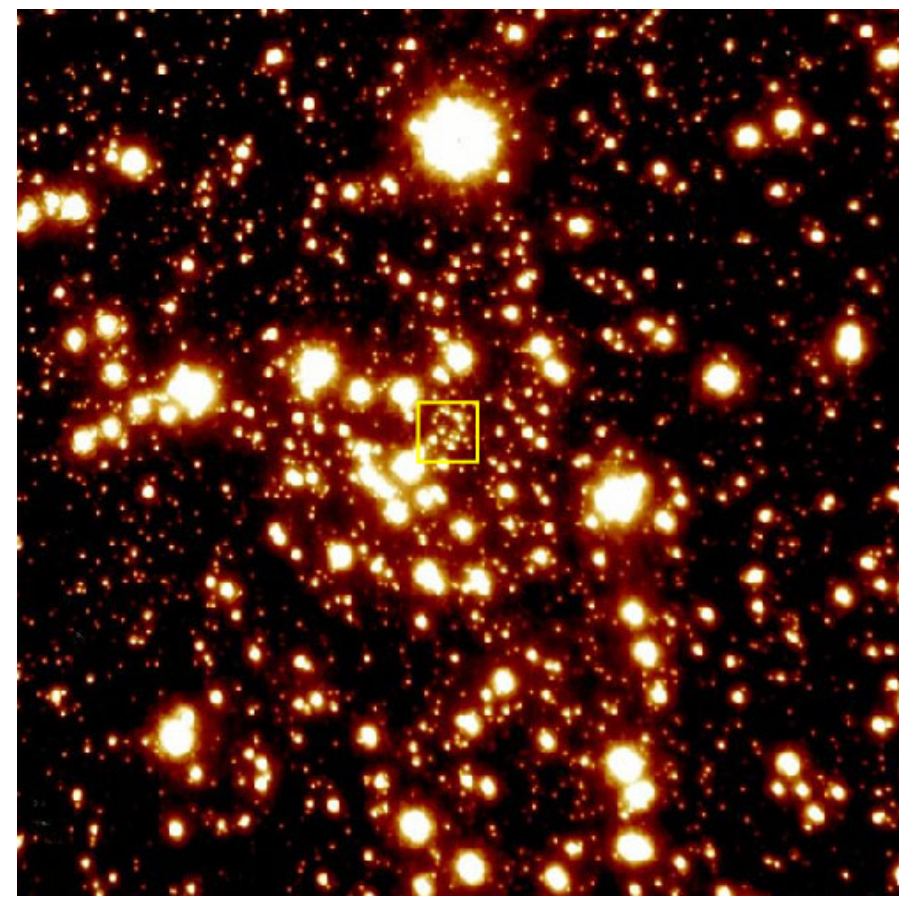

Figure 3. K-band image of the Central cluster obtained with NAOS/CONICA from Schödel et al. (2007). The 100 or so brightest stars in the image are evolved descendants from main sequence O-stars. The central box highlights the "s" stars that are presumably young and massive $\left(M_{\text {initial }} \approx 20 \mathrm{M}_{\odot}\right)$.

\subsection{Arches cluster}

The Arches cluster is the densest young cluster in the Galaxy, and it has a relatively young age (Figer et al. 2002). Being so young and massive, it contains the richest collection of O-stars and WNL stars in any cluster in the Galaxy (Harris et al. 1994; Nagata et al. 1995; Figer 1995; Cotera 1995; Cotera et al. 1996; Serabyn, Shupe, \& Figer 1998; Figer et al. 1999b; Blum et al. 2001; Figer et al. 2002; Figer 2005). The WNL stars are particularly interesting, as they represent the largest collection of the most massive stars $\left(\mathrm{M}>100 \mathrm{M}_{\odot}\right)$ in the Galaxy. As seen elsewhere, e.g. R136 and NGC 3603, these stars are still burning hydrogen on the main sequence. Given its unique combination of characteristics, the cluster is ideal as a testbed for measuring the upper mass cutoff to the IMF (see Section 3). The strong stellar winds from the most massive stars in the cluster are detected at radio wavelengths (Lang, Goss, \& Rodríguez 2001; Yusef-Zadeh et al. 2003; Lang et al. 2005; Figer et al. 2002), and x-ray wavelengths (Yusef-Zadeh et al. 2002; Rockefeller et al. 2005; Wang, Dong, \& Lang 2006).

\subsection{Quintuplet cluster}

The Quintuplet cluster was originally noted for its five very bright stars (Glass, Moneti, \& Moorwood 1990; Okuda et al. 1990; Nagata et al. 1990), but is now known to contain many massive stars (Geballe et al. 1994; Figer, McLean, \& Morris 1995; Timmermann et al. 1996; Figer et al. 1999a). It is $\approx 4$ Myr old and had an initial mass of $>10^{4} \mathrm{M}_{\odot}$ (Figer et al. 1999a). The hot stars in the cluster ionize the nearby "Sickle" HII region (see Figure 2). The Quintuplet is most similar to Westerlund 1 in mass, age, and spectral content. Some of the stars in the cluster have been detected at x-ray wavelengths (Law \& 
Yusef-Zadeh 2004), and at radio wavelengths (Lang et al. 1999, 2005). Recently, Tuthill et al. (2006) convincingly show that the five red stars in the cluster are dusty WC stars, characteristic of binary systems containing WCL plus an OB star (Tuthill, Monnier, \& Danchi 1999). This may indicate that either the binary fraction for massive stars is extremely high (Nelan et al. 2004), or only binary massive stars evolve through the WCL phase (van der Hucht 2001). The Quintuplet cluster also contains two Luminous Blue Variables, the Pistol star (Harris et al. 1994; Figer et al. 1998, 1999c), and FMM362 (Figer et al. 1999a; Geballe, Najarro, \& Figer 2000). Both stars are extraordinarily luminous $\left(L>10^{6} \mathrm{~L}_{\odot}\right)$, yet relatively cool $\left(T \approx 10^{4} \mathrm{~K}\right)$, placing them in the "forbidden zone" of the Hertzsprung-Russell Diagram, above the Humphreys-Davidson limit (Humphreys \& Davidson 1994). They are also both confirmed photometric and spectroscopic variables (Figer et al. 1999a). The Pistol star is particularly intriguing, in that it is surrounded by one of the most massive $\left(10 \mathrm{M}_{\odot}\right)$ circumstellar ejecta in the Galaxy (Figer et al. $1999 \mathrm{c}$; Smith 2008). Both stars are spectroscopically (Figer et al. 1999a) and photometrically variable (Glass et al. 2001), as expected for LBVs.

\section{An upper limit to the masses of stars}

Massive star clusters can be useful testing grounds for a variety of theoretical predictions, e.g. the IMF, binary fraction, n-body interactions, etc. Although only recently discovered, the currently known set of Galactic massive clusters have already yielded an interesting result regarding the upper limit to the masses of stars.

Theoretically, one might expect stellar mass to be limited by pulsational instabilities (Schwarzschild \& Härm 1959) or radiation pressure (Wolfire \& Cassinelli 1987). Although stellar evolution models have been computed for massive stars up masses of $1000 \mathrm{M}_{\odot}$, no such stars have ever been observed. Indeed, some of the most famous "massive stars" have turned out to be multiple systems.

Observationally, the problem is difficult because massive stars are rare. They are formed in small numbers with respect to lower mass stars and they only live a few million years. A star formation event must produce about $10^{4} \mathrm{M}_{\odot}$ in stars to have a statistically meaningful expectation of stars with masses greater than $150 \mathrm{M}_{\odot}$. For example, such a cluster should have about three such stars, assuming a Salpeter initial mass function (Salpeter 1955) extrapolated to zero probability. Unfortunately, the cluster must satisfy a number of other criteria in order to use it for identifying an upper mass cutoff. For instance, it must be young enough so that its most massive members have not yet exploded as supernovae. Yet, it must be old enough to be free of natal molecular material. In order to identify its individual stars, it must be close enough to us. To relate apparent magnitude to absolute magnitude, it must be at known distance. The stars must be coeval enough so that the star formation episode that produced the cluster can be considered to constitute a single event. Finally, the age must be known relatively accurately.

It is difficult to satisfy all these criteria, and, indeed, only the Arches cluster does. Being in the Galactic center happens to be useful in this regard, as its distance is then relatively well known as compared to other clusters. The result for the Arches cluster is shown in Figure 4. In this plot, it is apparent that there is an absence of stars with masses above about $150 \mathrm{M}_{\odot}$, where many are expected, i.e. there is an upper mass cutoff. See Weidner \& Kroupa (2004), Weidner \& Kroupa (2006) and Oey \& Clarke (2005) for other arguments for such a cutoff. 


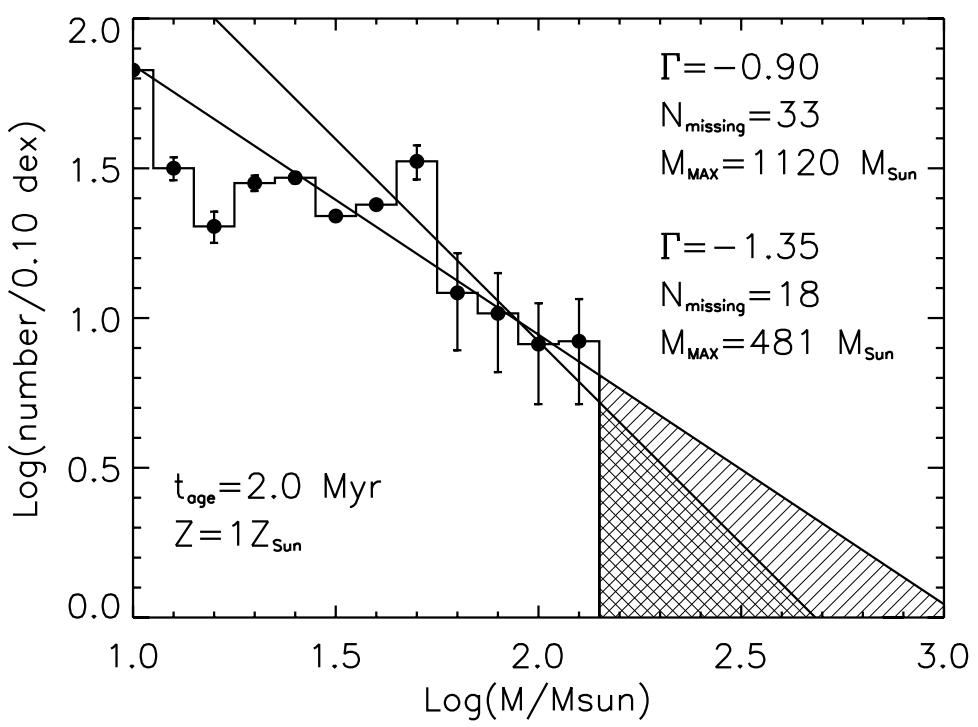

Figure 4. Number versus mass for stars in the Arches cluster from Figer (2005). There is a clear deficit of stars with initial masses greater than $150 \mathrm{M}_{\odot}$, as seen in the hatched regions, for a reasonable range of IMF slopes.

\section{The hidden population of massive stellar clusters}

2MASS (Skrutskie et al. 1997) and GLIMPSE (Benjamin et al. 2003) have heralded a new era in massive star cluster research. With these surveys, we will be able to probe much further into the Galactic plane than ever before. We can expect an order of magnitude increase in the number of known young clusters in the Galaxy as these surveys are further investigated. Figure 5 shows the Galactic distribution of known young clusters from WEBDA (dots), candidate clusters from Bica et al. (2003) (triangles), and the verified massive clusters discussed in this paper, with the addition of several very likely massive clusters (hexagons). The Galactic center is at $(0,0)$ and the Sun is at $(0,8)$. The visual clusters are mostly within $3 \mathrm{kpc}$ from the Sun. The infrared clusters are a bit further away; however, it is clear that the number is highly incomplete and that the far side of the Galaxy as well as the central regions must contain many new clusters yet to be discovered. We estimate that the total number of Galactic stellar clusters should exceed 20,000 (poster by Messineo, this volume).

\section{Acknowledgement}

I thank the following individuals for discussions related to this work: Ben Davies, Paco Najarro, Rolf Kudritzki, Maria Messineo, Lucy Hadfield, Qingfeng Zhu, and Sungsoo Kim. The material in this paper is based upon work supported by NASA under award No. NNG05-GC37G, through the Long Term Space Astrophysics program. This research has made use of the SIMBAD database, Aladin and IDL software packages, and the GSFC IDL library. This research was performed in the Rochester Imaging Detector Laboratory with support from a NYSTAR Faculty Development Program grant. Some of the data presented herein were obtained at the W. M. Keck Observatory, which is operated as a scientific partnership among the California Institute of Technology, the University of 


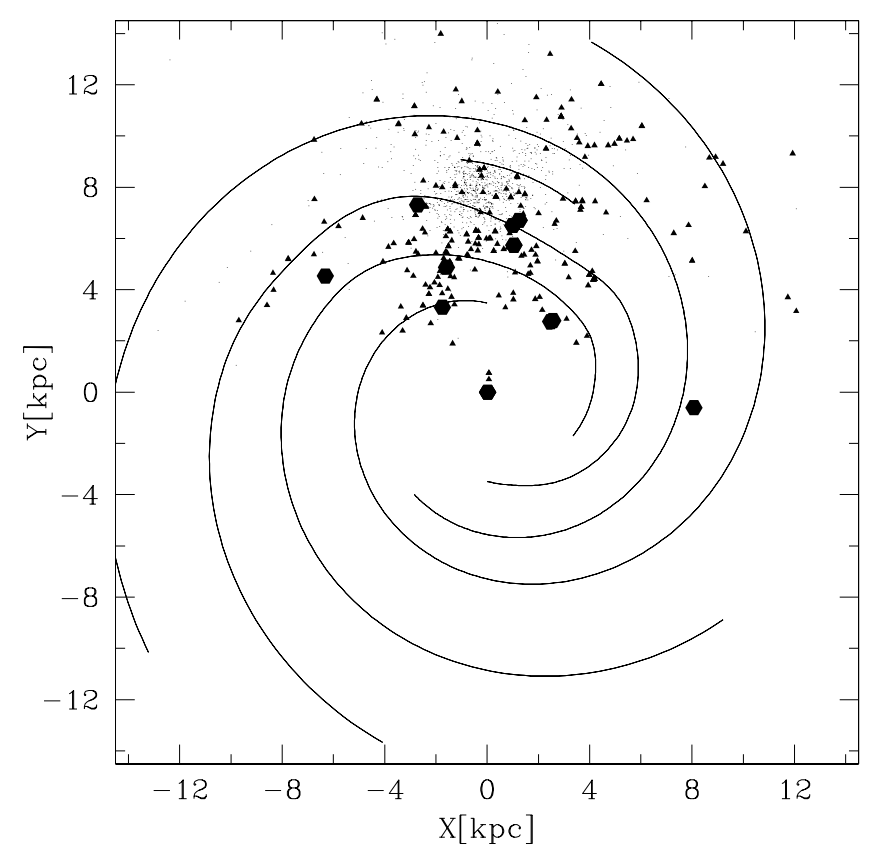

Figure 5. Galactic distribution of known young clusters (dots), candidate clusters (triangles), and verified massive clusters (hexagons). The Galactic center is at $(0,0)$ and the Sun is at $(0,8)$. Distance for the known young clusters are from Dias et al. (2002). Distances for the candidate clusters were determined by fitting the line-of-sight velocities of nearby HII regions (Kuchar \& Bania 1994) to the Galactic rotation curve, assuming that these regions are associated with the clusters. This figure is courtesy of Maria Messineo.

California, and the National Aeronautics and Space Administration. The Observatory was made possible by the generous financial support of the W. M. Keck Foundation.

\section{References}

Allen, D. A., Hyland, A. R., \& Hillier, D. J. 1990, MNRAS, 244, 706

Barbosa, C. \& Figer, D. 2004, (arXiv:astro-ph/0408491)

Becklin, E. E., Matthews, K., Neugebauer, G., \& Willner, S. P. 1978, ApJ, 219, 121

Benjamin, R. A., Churchwell, E., Babler, B. L. et al. 2003, PASP, 115, 953

Bica, E., Dutra, C. M., Soares, J., \& Barbuy, B. 2003, A\&A, 404, 223

Blum, R. D., Schaerer, D., Pasquali A. et al. 2001, AJ, 122, 1875

Blum, R. D., Sellgren, K., \& Depoy, D. L. 1995b, ApJ, 440, L17

Blum, R. D., Sellgren, K., \& Depoy, D. L. 1996a, AJ, 112, 1988

Brandner, W., Clark, J. S., Stolte, A. et al. 2008, A\& A, 478, 137

Clark, J. S., Negueruela, I., Crowther, P. A., \& Goodwin, S. P. 2005, A\&A, 434, 949

Cotera, A. S. 1995, Ph.D. Thesis

Cotera, A. S., Erickson E. F., Colgan, S. W.J. et al. 1996, ApJ, 461, 750

Cotera, A. S., Simpson, J. P., Erickson E. F. et al. 1999, ApJ, 510, 747

Crowther, P. A., Hadfield, L. J., Clark, J. S. et al. 2006, MNRAS, 372, 1407

Davies, B., Figer D. F., Kudritzki, R.-P. et al. 2007, ApJ, 671, 781

Davies, B., Figer D. F., Law, C. J. et al. 2008, ApJ, 676, 1016

Dias, W. S., Alessi, B. S., Moitinho, A., \& Lépine, J. R. D. 2002, A\& A, 389, 871

Eisenhauer, F., Genzel, R., Alexander, T. et al. 2005, ApJ, 628, 246 
Figer, D. F. 1995, Ph.D. Thesis

Figer, D. F. 2005, Nature, 434, 192

Figer, D. F. 2008, in press (arXiv:0803.1619)

Figer, D. F., Najarro, F., Gilmore, D. et al. 2002, ApJ, 581, 258

Figer, D. F., Kim, S. S., Morris, M. et al. 1999b, ApJ, 525, 750.

Figer, D. F., MacKenty, J. W., Robberto, M. et al. 2006, ApJ, 643, 1166

Figer, D. F., McLean, I. S., \& Morris, M. 1995, ApJ, 447, L29

Figer, D. F., McLean, I. S., \& Morris, M. 1999a, ApJ, 514, 202

Figer, D. F., Morris, M., Geballe, T. R. et al. 1999c, ApJ, 525, 759

Figer, D. F., Najrro, F., Geballe, T. R. et al. 2005, ApJ, 622, L49

Figer, D. F., Najarro, F., Morris, M. et al. 1998, ApJ, 506, 384

Forrest, W. J., Shure, M. A., Pipher, J. L., \& Woodward, C. E. 1987, in: D. C. Backer (ed.), The Galactic Center (New York: AIP), AIP Conf Proc 155, 153

Geballe, T. R., Genzel, R., Krabbe, A. et al. 1994, in: I. S. McLean (ed.), Infrared Astronomy with Arrays, The Next Generation, Astrophys Space. Sci. Lib. 190, 73

Geballe, T. R., Najarro, F., \& Figer, D. F. 2000, ApJ, 530, L97

Genzel, R., Schödel, R., Ott, T. et al. 2003, ApJ, 594, 812

Genzel, R., Thatte, N., Krabbe A. et al. 1996, ApJ, 472, 153

Glass, I. S., Matsumoto, S., Carter, B. S., \& Sekiguchi, K. 2001, MNRAS, 321, 77

Glass, I. S., Moneti, A., \& Moorwood, A. F. M. 1990, $M N R A S, 242,55 \mathrm{P}$

Groh, J. H., Damineli, A., Teodoro, M., \& Barbosa, C. L. 2006, A\& A, 457, 591

Harayama, Y. 2007, PhD Thesis, LMU München

Harris, A. I., Krenz, T., Genzel, R. et al. 1994, in: R. Genzel \& A. I. Harries (eds.), The Nuclei of Normal Galaxies: Lessons from the Galactic Center, (Dodrecht: Kluwer), NATO Advanced Science Inst. Series C, 445, 223

Humphreys, R. M., \& Davidson, K. 1994, PASP, 106, 1025

Kim, S. S., Figer, D. F., Kudritzki, R. P., \& Najarro, F. 2006, ApJ, 653, L113

Krabbe, A., et al. 1995, ApJ, 447, L95

Krabbe, A., Genzel, R., Drapatz, S., \& Rotaciuc, V. 1991, ApJ, 382, L19

Kuchar, T. A., \& Bania, T. M. 1994, ApJ, 436, 117

Lang, C. C., Figer, D. F., Goss, W. M., \& Morris, M. 1999, AJ, 118, 2327

Lang, C. C., Goss, W. M., \& Rodríguez, L. F. 2001, ApJ, 551, L143

Lang, C. C., Johnson, K. E., Goss, W. M., \& Rodríguez, L. F. 2005, AJ, 130, 2185

Law, C. \& Yusef-Zadeh, F. 2004, ApJ, 611, 858

Lebofsky, M. J., Rieke, G. H., \& Tokunaga, A. T. 1982, ApJ, 263, 736

Muno, M. P., Clark, J. S., Crowther P. A. et al. 2006, ApJ, 636, L41

Nagata, T., Woodward, C. E., Shure, M., \& Kobayashi, N. 1995, AJ, 109, 1676

Nagata, T., Woodward, C. E., Shure, M. et al. 1990, ApJ, 351, 83

Najarro, F. 1995, Ph.D. Thesis

Najarro, F., Hillier, D. J., Kudritzki, R.-P. et al. 1994, A\&A, 285, 573

Najarro, F., Krabbe, A. Genzel, R. et al. 1997, A\&A, 325, 700

Nakaya, H., Watanabe, M., Ando, M. et al. 2001, AJ, 122, 876

Negueruela, I. \& Clark, J. S. 2005, A\&A, 436, 541

Nelan, E. P., Walborn, N. R., Wallace, D. J. et al. 2004, AJ, 128, 323

Oey, M. S. \& Clarke, C. J. 2005, ApJ, 620, L43

Okuda, H., Shibai, H., Nakagawa, T. et al. 1990, ApJ, 351, 89

Rieke, G. H., Telesco, C. M., \& Harper, D. A. 1978, ApJ, 220, 556

Rockefeller, G., Fryer, C. L., Melia, F., \& Wang, Q. D. 2005, ApJ, 623, 171

Salpeter, E. E. 1955, ApJ, 121, 161

Schwarzschild, M. \& Härm, R. 1959, ApJ, 129, 637

Schödel, R., Eckart, A., Alexander, T. et al. 2007, A\&SA, 469, 125

Serabyn, E., Shupe, D., \& Figer, D. F. 1998, Nature, 394, 448

Skinner, S. L., Simmons, A. E., Zhekov, S. A. et al. 2006, ApJ, 639, L35 
Skrutskie, M. F., Schneider, S. E., Stiening, R. et al. 1997, in: F. Garzon (ed.), The Impact of Large Scale Near-IR Sky Surveys (Dordrecht: Kluwer), Astrophys Space Sci Lib, 210, 25

Smith, N. 2008, in: M. Livio (ed.), Massive Stars: From Pop III and GRBs to the Milky Way, (Cambridge: CUP) in press (arXiv:astro-ph/0607457)

Stephenson, C. B. 1990, AJ, 99, 1867

Tanner, A., Figer, D. F., Najarro, F. et al. 2006, ApJ, 641, 891

Timmermann, R., Genzel, R., Poglitsch, A. et al. 1996, ApJ, 466, 242

Tuthill, P. G., Monnier, J. D., \& Danchi, W. C. 1999, Nature, 398, 487

Tuthill, P., Monnier, J., Tanner, A. et al. 2006, Science, 313, 935.

van der Hucht, K. A. 2001, New Astronomy Review, 45, 135

Wang, Q. D., Dong, H., \& Lang, C. 2006, MNRAS, 371, 38

Weidner, C. \& Kroupa, P. 2004, MNRAS, 348, 187

Weidner, C. \& Kroupa, P. 2006, MNRAS, 365, 1333

Wolfire, M. G. \& Cassinelli, J. P. 1987, ApJ, 319, 850

Yusef-Zadeh, F., Law, C., Wardle M. et al. 2002, ApJ, 570, 665

Yusef-Zadeh, F. \& Morris, M. 1987, ApJ, 320, 545

Yusef-Zadeh, F., Nord, M., Wardle, M. et al. 2003, ApJ, 590, L103

\section{Discussion}

VANBEVEREN: In 1982, I published a study where it was shown (from a theoretical point of view) that the maximum mass of the stars in a cluster depends on the total mass of the cluster and, from the cluster (total) mass function, I proposed a mass function for the maximum mass. This idea has been picked up by Weidner and Kroupa in 2004. Is there any observational evidence that he maximum mass of the stars in a cluster depends on the cluster mass?

FIGER: Some work to answer that question has been done by Oey \& Clarke (2005); however, the clusters in that study have very uncertain ages and it is thus not clear if their most massive members have already exploded as supernovae.

DAMINELI: Your open cluster candidates do not fit the "radii" portion of the arms, specifically in the Carina arm. How did you assign distances to the clusters?

FIGER: We considered clusters associated with HII regions for which the line-of-velocities are available (Kuchar \& Bania 1994). We therefore obtained distances by using the equations in Burton (1988), assuming near-distances.

LANGER: You use the argument that no WNE or WC stars are found in the Arches cluster to prove that it is young enough so that the most massive stars have not exploded off yet. Is this challenged by the evidence put forward by Nathan Smith that the most massive stars die as hydrogen-rich LBVs?

FIGER: Nathan Smith's speculation suggests that the most massive stars die as WNh. If this is true, then it is possible that we can no longer see stars with initial masses above $150 \mathrm{M}_{\odot}$ in the Arches cluster because they have already exploded as supernovae, NOT because they did not form. This would also be true for R136 in 30 Dor. One interesting observational test of such a claim is to search for coeval clusters in which there are simultaneously WNh stars and more evolved subtypes (presumably from the highest initial masses in the cluster). Perhaps NGC 3603 is promising for such a test where there are WNh stars and a more evolved subtype, Sher 25; however, one would have to clearly show that these stars are coeval. 